\section{In search for the ideal anatomical composition of vascularised human skin equivalents for systemic sclerosis translational research: should we recruit the telocytes?}

The recent paper by Matei et $a l^{1}$ has raised our interest on the feasibility and reliability of vascularised human skin equivalents for fibrosis research. This is a novel in vitro model which may replicate key features of fibrotic skin and may become a valuable platform for preclinical testing of innovative therapeutic strategies for systemic sclerosis (SSc) and other cutaneous fibrosing conditions. In this informative study, an engineered human skin equivalent featuring a functional vascular system with physiological perfusion was established by sequential seeding of primary human endothelial cells, fibroblasts and keratinocytes on a three-dimensional (3D) extracellular matrix scaffold. ${ }^{1}$ Elegantly, the authors have shown that their 3D system may reproduce the main features of human skin relevant for the pathogenesis of skin fibrosis. Indeed, the exposure of these vascularised human skin equivalents to profibrotic transforming growth factor- $\beta$ (TGF $\beta$ ) has induced the fibroblast-to-myofibroblast transition and abnormal deposition of extracellular matrix, thus closely mimicking the SSc skin microenvironment. ${ }^{1}$ Moreover, the induction of dermal fibrosis was efficiently prevented by nintedanib, a tyrosine kinase inhibitor with proven antifibrotic effects, which further demonstrated that this model might serve as a suitable test system for future targeted therapies. The authors have also clearly discussed how their innovative model may help in overcoming many limitations currently encountered by scientists engaged in SSc translational research when using classical two-dimensional cell culture systems and in vivo mouse models. $^{1}$

Overall, we are confident that this pioneer work $^{1}$ will represent the necessary groundwork for further studies devoted to refining the $3 \mathrm{D}$ composition of vascularised human skin equivalent systems. This effort may increase even more their similarity to the microscopic anatomical structure of human skin and some interesting cues should be considered. As pointed out by the authors, a strength of their $3 \mathrm{D}$ model system is that it may replicate the relevant cell-matrix interactions, the crosstalk between different skin cell types and the signalling pathways related to these processes. ${ }^{1}$ Usually, these events are crucial for the physiological maintenance of tissue homeostasis and their modification/impairment may trigger the development of skin fibrosis. However, a 3D system including only endothelial cells, fibroblasts and keratinocytes clearly cannot fully recapitulate the human skin environment either in a physiological or in a profibrotic condition. In fact, other cells resident in the skin may have a role in the fibrotic progression of SSc, including TGF $\beta$-secreting mast cells, as well as pericytes located in the microvessel wall and preadipocytes, found in the adipose-derived stromal vascular fraction, that may be a possible source of profibrotic myofibroblasts. ${ }^{2} 3$ Furthermore, it appears that fibroblast heterogeneity may be crucial in determining dermal architecture during skin development and repair. ${ }^{45}$ Recently, it has been shown that skin fibroblasts arise from two distinct lineages. ${ }^{5}$ The first one forms the upper dermis, including the dermal papilla regulating hair growth and the arrector pili muscle. ${ }^{5}$ The second one instead forms the lower dermis and the hypodermis, including the reticular fibroblasts synthesising the bulk of the fibrillar extracellular matrix and the preadipocytes/adipocytes. ${ }^{5}$ Ideally, these relevant different fibroblast subpopulations should therefore be
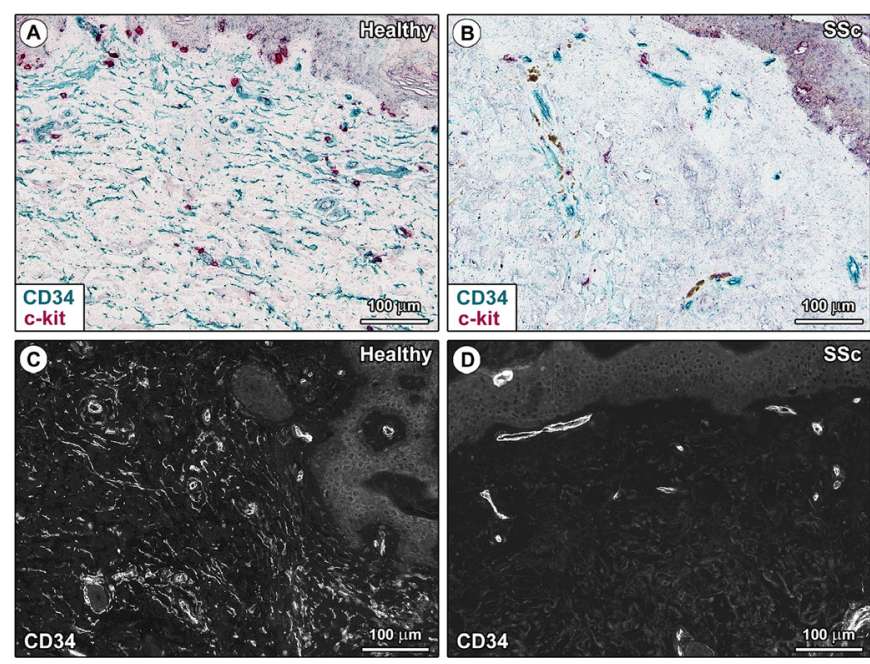

Figure 1 The arrangement of telocytes/CD34-positive stromal cells in healthy and systemic sclerosis (SSC) skin. (A and B) Representative microphotographs of skin sections subjected to immunoperoxidasebased immunohistochemistry for CD34 (green) and c-kit (red). An extensive network of telocytes/CD34-positive stromal cells is evident throughout the whole dermis of healthy skin $(A)$, while telocytes are almost undetectable in advanced/fibrotic SSc skin (B). In both healthy and SSc skin, vascular structures are CD34-positive, while mast cells are c-kit-positive. (C and D) Representative microphotographs of skin sections subjected to CD34 immunofluorescence staining. (C) Note the complex network formed by telocytes/CD34-positive stromal cells in healthy dermis. (D) No telocyte/CD34-positive stromal cell can be detected in advanced/fibrotic SSc dermis.

represented in primary fibroblast cultures employed to establish human skin equivalents for translational research purposes.

In this scenario, we believe it would be worth to focus the attention on a peculiar stromal cell population that has been recently identified in human skin and other tissues/organs, the telocytes. ${ }^{6}$ Telocytes, also referred to as CD34-positive stromal cells, possess extremely long prolongations with distinct ultrastructural features (telopodes). ${ }^{6}$ These cells form a complex 3D stromal meshwork establishing a multitude of intercellular contacts with a variety of cell types. ${ }^{6}$ The peculiar morphology, spatial distribution and ability to release different kinds of extracellular vesicles make telocytes increasingly interesting regulators of intercellular signalling in the coordination of tissue morphogenesis during development and maintenance of local tissue homeostasis in post-natal life. ${ }^{6}$ In addition, structural changes in telocyte networks have been recently reported in different disorders. ${ }^{7}$ In the skin in physiologic conditions, telocytes constitute an extensive scaffold-like cellular network which compartmentalises the dermal connective tissue (figure 1). ${ }^{8}$ In both the upper papillary and lower reticular dermis, telocyets intimately surround the vessels and the skin adnexa and establish numerous intercellular communications with neighbour cell types, such as fibroblasts and mast cells (figure 1). ${ }^{9}$ Recently, we have shown that clinically involved SSc skin displays a progressive disruption of the dermal network of telocytes up to almost their complete loss in the advanced/fibrotic cutaneous disease stage (figure 1). ${ }^{9}$ In SSc, the impairment of the telocyte network has also been shown in fibrotic lesions of the lung, the myocardium and the gastric wall. ${ }^{10}$ On this evidence, we suggested that the telocyte loss might play a relevant role in SSc pathogenesis by favouring the dysregulation of intercellular signalling mechanisms that control the fibroblast/myofibroblast activity and contributing 
to the progressive loss of the normal tissue structure. ${ }^{9}{ }^{10}$ At the moment, the protocols for selective telocyte purification are still at an early stage and more work is needed to definitively establish primary cultures. When this will be, telocytes cultured in vitro could represent an added value to improve the reliably building of vascularised human skin equivalents as suitable models for SSc translational research.

\section{Mirko Manetti $\odot,{ }^{1}$ Marco Matucci-Cerinic ${ }^{2}$}

${ }^{1}$ Department of Experimental and Clinical Medicine, Section of Anatomy and Histology, University of Florence, Florence, Italy

${ }^{2}$ Department of Experimental and Clinical Medicine, Section of Rheumatology, University of Florence, Florence, Italy

Correspondence to Dr Mirko Manetti, Department of Experimental and Clinical Medicine, Section of Anatomy and Histology, University of Florence, Florence 50134, Italy; mirko.manetti@unifi.it

Contributors $\mathrm{MM}$ and $\mathrm{MM}-\mathrm{C}$ have completely drafted and finalised the manuscript.

Funding The authors have not declared a specific grant for this research from any funding agency in the public, commercial or not-for-profit sectors.

Competing interests None declared.

Patient consent for publication Not required.

Provenance and peer review Not commissioned; internally peer reviewed. (c) Author(s) (or their employer(s)) 2019. No commercial re-use. See rights and permissions. Published by BMJ.

Check for updates
To cite Manetti M, Matucci-Cerinic M. Ann Rheum Dis Epub ahead of print: [please include Day Month Year]. doi:10.1136/annrheumdis-2019-216371

Received 25 September 2019

Accepted 26 September 2019

Ann Rheum Dis 2019;0:1-2. doi:10.1136/annrheumdis-2019-216371

ORCID iD

Mirko Manetti http://orcid.org/0000-0003-3956-8480

\section{REFERENCES}

1 Matei A-E, Chen C-W, Kiesewetter L, et al. Vascularised human skin equivalents as a novel in vitro model of skin fibrosis and platform for testing of antifibrotic drugs. Ann Rheum Dis 2019. doi:10.1136/annrheumdis-2019-216108. [Epub ahead of print: 20 Sep 2019].

2 Ebmeier S, Horsley V. Origin of fibrosing cells in systemic sclerosis. Curr Opin Rheumatol 2015;27:555-62.

3 Hügle T, Hogan V, White KE, et al. Mast cells are a source of transforming growth factor $\beta$ in systemic sclerosis. Arthritis Rheum 2011;63:795-9.

4 Garrett SM, Baker Frost D, Feghali-Bostwick C. The mighty fibroblast and its utility in scleroderma research. J Scleroderma Relat Disord 2017;2:100-7.

5 Driskell RR, Watt FM. Understanding fibroblast heterogeneity in the skin. Trends Cell Biol 2015;25:92-9.

6 Cretoiu SM, Popescu LM. Telocytes revisited. Biomol Concepts 2014;5:353-69.

7 Ibba-Manneschi L, Rosa I, Manetti M. Telocytes in chronic inflammatory and fibrotic diseases. Adv Exp Med Biol 2016;913:51-76.

8 Kang Y, Zhu Z, Zheng Y, et al. Skin telocytes versus fibroblasts: two distinct dermal cell populations. J Cell Mol Med 2015:19:2530-9.

9 Manetti M, Guiducci S, Ruffo M, et al. Evidence for progressive reduction and loss of telocytes in the dermal cellular network of systemic sclerosis. J Cell Mol Med 2013;17:482-96.

10 Manetti M, Rosa I, Messerini L, et al. A loss of telocytes accompanies fibrosis of multiple organs in systemic sclerosis. J Cell Mol Med 2014;18:253-62. 